\title{
Successful carbon-ion radiotherapy for choroidal melanoma adjacent to the optic disc: A case report
}

\author{
Masaya Taniguchi* \\ Taniguchi Eye Clinic, Aichi, Japan
}

\begin{abstract}
This report presents the case of a patient with choroidal melanoma adjacent to the optic disc, who was successfully treated with carbon-ion radiotherapy. A 44-yearold woman presented with a two-week history of blurred vision in her left eye. Before treatment, her best-corrected visual acuity was 1.2 and intraocular pressure (IOP) was $16 \mathrm{mmHg}$ in the left eye. Funduscopic examination revealed a choroidal mass located nasally adjacent to the optic nerve. B-mode echography and magnetic resonance imaging (MRI) showed an intraocular mass, approximately $4 \mathrm{~mm}$ thick. The patient was diagnosed with choroidal melanoma and underwent carbon-ion radiotherapy with an irradiation dosage of $70 \mathrm{~Gy}$. During the two-year follow-up, her best-corrected visual acuity was 1.0 and IOP was $15 \mathrm{mmHg}$ in the left eye. Slitlamp examination showed no neovascularization in the iris. Funduscopic examination revealed cicatrization of the tumor. B-mode echography and MRI revealed slight decrease in the tumor thickness. Systemic computed tomographic imaging did not reveal any suspicious metastatic lesion.
\end{abstract}

\section{Introduction}

Choroidal melanoma is a rare but life-threatening intraocular malignant tumor [1]. Local control of tumors without ocular complications and prevention of metastasis are the principal modalities for the management of choroidal melanoma.

Eye-preserving therapeutic modalities are available for choroidal melanoma [1]. However, since damage to the optic nerve cannot be avoided after eye-preserving therapy in patients with choroidal melanoma adjacent to the optic nerve, enucleation is commonly selected for such patients [2].

Carbon-ion radiotherapy is a recently established radiotherapy with unique physiological and biological properties that provide welllocalized, high-linear energy transfer [3]. Therefore, this therapy is effective and leads to minimum damage in the surrounding tissues [3]. Herein, I report the case of a patient with choroidal melanoma adjacent to the optic disc who was successfully treated with carbon-ion radiotherapy without vision loss.

\section{Case report}

Written informed consent was obtained from the patient for the publication of this case report and any accompanying images. A 44-year-old woman presented with a two-week history of blurred vision in her left eye. The patient did not have any prior or family history.

Before treatment, her best-corrected visual acuity was $1.2 \mathrm{OU}$ and the intraocular pressure (IOP) was $15 \mathrm{mmHg}$ OD and $16 \mathrm{mmHg}$ OS. Slitlamp examination revealed no remarkable changes. Funduscopic examination showed a choroidal mass nasally adjacent to the optic nerve with serous retinal detachment in the lesion (Figure 1A). B-mode echography revealed a $4.9 \mathrm{~mm}$ thick intraocular mass (Figure 1B). T1and T2-weighted magnetic resonance imaging (MRI) showed a hyperand hypo-intense intraocular mass (thickness, $4 \mathrm{~mm}$ and maximum diameter, $10 \mathrm{~mm}$ ) nasally adjacent to the optic nerve (Figure 1C). No extraocular invasion was found. The intraocular mass was diagnosed as a medium-sized choroidal melanoma, defined by the Collaborative Ocular Melanoma Study [4]. Positron emission tomography did not show any systemic hot spot.

The patient underwent carbon-ion radiotherapy (Figure 1D). Doses of carbon-ion beams were delivered at $70 \mathrm{~Gy}$ in 5 fractions with 1 anterior port. The patient was directed to gaze in the left direction during radiotherapy.

At the 2-year follow-up period, the patient's best-corrected visual acuity was 1.0 and IOP was $13 \mathrm{mmHg}$ in the left eye. The critical flicker frequency, which decreases with optic nerve damage, was 35 $\mathrm{Hz}$ in the left eye (normal: $\geq 35 \mathrm{~Hz}$ ). Slitlamp examination showed no neovascularization in the iris. Funduscopic examination revealed cicatrization of the tumor (Figure 1E). B-mode echography and MRI revealed a decrease in tumor thickness (Figure $1 \mathrm{~F}$ and $1 \mathrm{G}$ ). Systemic computed tomographic images did not show any suspicious metastatic lesions.

\section{Discussion}

This report details the case of a patient with choroidal melanoma adjacent to the optic disc who was successfully treated with carbon-ion radiotherapy.

The tumor did not systemically metastasize in this patient. Although contact between a tumor and the optic disc could increase the risk of metastasis [5], the tumor adjacent to the optic disc caused blurred vision, which enabled relatively early detection and treatment of the tumor, thereby preventing systemic metastasis in this patient.

Correspondence to: Masaya Taniguchi, MD, Taniguchi Eye Clinic, 5-50 Chiyogaoka, Chikusa, Nagoya, Aichi 464-0005, Japan, Tel: +81-52-777-6600; Fax: +81-52-777-6600; E-mail: mtani@m9.dion.ne.jp

Key words: choroidal melanoma, carbon-ion radiotherapy, optic disc

Received: February 01, 2017; Accepted: February 17, 2017; Published: February 20, 2017 


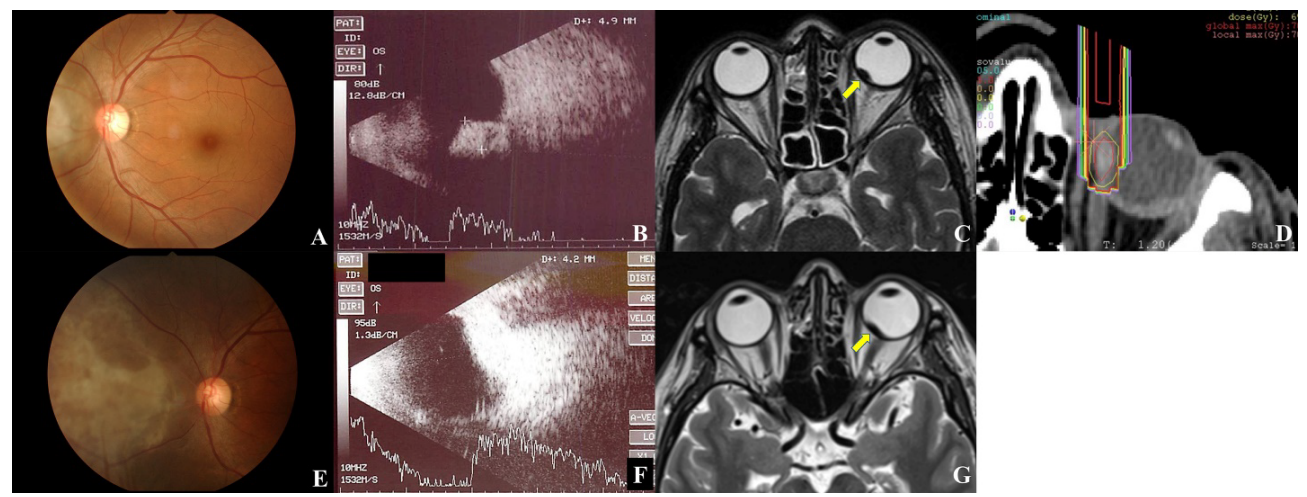

Figure 1. Clinical images. A. A pre-treatment funduscopic image showing a choroidal tumor located nasally adjacent to the optic disc. B. Pre-treatment B-mode echography showing a $4.9 \mathrm{~mm}$ thick intraocular mass. C. A pre-treatment T2-weighted axial magnetic resonance image (MRI) showing a hypo-intense intraocular mass located nasally adjacent to the optic disc (arrow). D. Dose distribution on a CT image for treatment planning. The iso-dose lines indicate $10 \%, 30 \%, 50 \%, 80 \%, 90 \%$, and $95 \%$ dose areas from the outside to the inside. E. A funduscopic image taken 2 years after treatment showing cicatrization of the tumor. F. B-mode echography taken 2 years after treatment showing decreased thickness of 4.2 mm. G. A T2weighted axial MRI taken 2 years after treatment showing slightly reduced tumor thickness (arrow).

The patient did not experience vision loss in the affected eye during the two-year post-radiotherapy period. Neovascular glaucoma (NVG) and ischemic optic neuropathy are major causes of vision loss after carbon-ion radiotherapy [6]. A large irradiated volume in the anterior segment and optic nerve was indicated to be the cause of intraocular ischemic changes [6]. However, the left gaze during radiotherapy may minimize irradiation to the anterior segment and optic nerve, resulting in neither NVG nor neuropathy in this patient.

Local recurrence, distant metastasis, and NVG can occur during the late follow-up period $[1,6]$. Therefore, continuous monitoring was required for this patient.

In conclusion, this report details the case of a patient with choroidal melanoma adjacent to the optic disc who was successfully treated with carbon-ion radiotherapy, thereby demonstrating the usefulness of this therapeutic modality for such patients.

\section{Acknowledgements}

The author wishes to thank Dr. Toshinobu Kubota from the Department of Ophthalmology, Nagoya Medical Center, and Dr. Hiroshi Tsuji from the Research Center Hospital for Charged Particle Therapy, National Institute of Radiological Sciences, for the collection of patient data.

\section{Conflicts of interest}

None.

\section{Disclosure of funding}

None.

\section{References}

1. Kaliki S, Chields CL (2017) Uveal melanoma: relatively rare but deadly cancer. Eye 31: 241-257. [Crossref]

2. Perry JD, Singh AD, Mehta MP (2013) Enucleation for choroidal melanomas. In: Ryan SJ, (ed.): Retina. Fifth Edition. Philadelphia: Elsevier Saunders 2: 2271-2274.

3. Tsuji M, Kimura K, Tsuji H, Goto M, Yoshikawa H, et al. (2007) Histological study of choroidal malignant melanoma treated by carbon ion radiotherapy. Jpn J Ophthalmol 51: 127-130. [Crossref]

4. Margo CE (2004) The Collaborative Ocular Melanoma Study: an overview. Cancer Control 11: 304-309. [Crossref]

5. Mizota A (2016) Carbon-ion charged particle therapy in ophthalmology. Rinsho Ganka 70: 1357-1363 (Japanese).

6. Toyama S, Tsuji H, Mizoguchi N, Nomiya T, Kamada T, et al. (2013) Long-term results of carbon ion radiation therapy for locally advanced or unfavorably located choroidal melanoma: usefulness of CT-based 2-port orthogonal therapy for reducing the incidence of neovascular glaucoma. Int J Radiat Oncol Biol Phys 86: 270-276. [Crossref]

Copyright: (C2017 Taniguchi M. This is an open-access article distributed under the terms of the Creative Commons Attribution License, which permits unrestricted use, distribution, and reproduction in any medium, provided the original author and source are credited. 